\title{
GNSS POSITIONING ALGORITHMS USING METHODS OF REFERENCE POINT INDICATORS
}

\author{
Bartlomiej Oszczak \\ Department of Satellite Geodesy and Navigation \\ Faculty of Geodesy and Land Management \\ University of Warmia and Mazury in Olsztyn, Poland \\ Department of Aircraft Navigation \\ Polish Air Force Academy in Deblin, Poland \\ b.oszczak@kgsin.pl
}

\begin{abstract}
The GNSS standard positioning solution determines the coordinates of the GNSS receiver and the receiver clock offset from measurements of at least four pseudoranges. For GNSS positioning, a direct solution was derived for five and ten observed satellites without linearisation of the observation equations and application of the least squares method. The article presents the basic principles of methods for solving the positioning problem, the formulas and their derivation. The numerical examples with simulated pseudorange data confirm the correct performance of the proposed algorithm. The presented algorithms should be further tested with real measurements in other domains of positioning and navigation as well.
\end{abstract}

Keywords- Global Positioning System, Satellite Navigation Systems, Radio Navigation, Navigation, Aircraft Navigation, Marine Navigation, Least Squares Approximation, Linear Approximation, Reference Point Indicator.

\section{INTRODUCTION}

\subsection{Standard GNSS Single Positioning Algorithms}

The method of point positioning with code ranges (Hoffman-Wellenhof, 2008) consists of determining the coordinates of the GNSS receiver antenna based on the known coordinates of at least four satellites and the measured pseudoranges corrected for ionospheric and tropospheric refraction using algorithms proposed by e.g. Klobuchar (1986) and Hopfield (1969). In this method, the subject of determination are the Cartesian coordinates of a GNSS antenna and the receiver clock error. After the introduction of these necessary corrections to the observed pseudoranges, the antenna position of point $Q\left(x_{Q}, y_{Q}, z_{Q}\right)$ in the ECEF coordinate system and the receiver clock bias $b_{o}$ can be determined by using the linearisation technique and least squares method (Strang and Borre, 1997). In a standard positioning algorithm, the iteration technique is used in which the known position of the GNSS receiver is approximated (Tsui, 2000). When values of the approximate position are not accurate enough, the Time To First Fix (TTFF) (Paonni et al., 2010) is expected to be longer, i.e. the time to start the navigation process is delayed. Many authors have discussed the different concepts of solving the positioning algorithm. The Bancroft algorithm (1991) consists of a $4 \times 4$ matrix 
inversion and the solution of scalar equations to the second degree. The algorithm has been widely discussed and analyzed by Abel and Chafee (1991) and Chafee and Abel (1991) Another solution given by Grafarend and Chan (1996) is based on the quadratic form of observation equations for algebraic reduction of numbers of observation equations. Kleusberg (1994) also discussed subsequent options for determining a unique solution among many others as well as geometrical conditions of various cases. In the article, it has been shown that a position can be determined for at least five measured pseudoranges without the need of the linearisation technique and the least squares method based on introduced definitions of reference point indicators with regard to a point to be determined.

\subsection{Definition of Fixed Point Indicator in Two Dimensional Space}

Fixed point indicator $t$ in surveying was defined by Hausbrandt (1970). From this definition, it follows that, quoting the author "for the indicator of the fixed point with regard to the determined point in two dimensional space, we can write the equation":

$$
t_{1}=x_{1}^{2}+y_{1}^{2}-d_{1}^{2}
$$

where: $x_{1}, y_{1}-$ plane coordinates of the fixed point $1\left(x_{1}, y_{1}\right)$,

$d_{1}$ - distance from the determined point to the fixed point $1\left(x_{1}, y_{1}\right)$.

For three fixed points $1\left(x_{1}, y_{1}\right), 2\left(x_{2}, y_{2},\right), 3\left(x_{3}, y_{3}\right)$ and distances $d_{1}, d_{2}, d_{3}$ the solution of linear plane resection is expressed by Hausbrandt's equation:

$$
\left[\begin{array}{ll}
x_{Q} & y_{Q}
\end{array}\right]=\frac{1}{2}\left[\begin{array}{ll}
\Delta t_{12} & \Delta t_{13}
\end{array}\right]\left[\begin{array}{ll}
\Delta x_{12} & \Delta y_{12} \\
\Delta x_{13} & \Delta y_{13}
\end{array}\right]^{-1}
$$

where:

$Q\left(x_{Q}, y_{Q}\right)$ - the coordinates of point $Q$ to be determined,

$\Delta t_{1 k}=t_{k}-t_{1}, k=2,3$

$\Delta x_{1 k}=x_{k}-x_{1}$

$\Delta y_{1 k}=y_{k}-y_{1}$

For each fixed point, its point indicator (1) is computed in regard to the determined point $Q\left(x_{Q}, y_{Q}\right)$ :

$$
t_{i}=x_{i}^{2}+y_{i}^{2}-d_{i}^{2}
$$

where $i=1,2,3$.

\subsection{Definition of Reference Point Indicator Proposed by the Author}

Hausbrandt defined the fixed point indicator (Hausbrandt, 1970) with regard to the determined point in 1970. For GNSS, a receiver position is computed in reference to the satellite positions (Misra and Enge, 2006), which are not fixed in the meaning of Hausbrant's definition of a fixed point indicator. Instead, they move in orbits and the GNSS constellation changes continuously. Thus "fixed point indicator" needs to be broadened with a new term. The author proposes reference point indicator $t_{i}$ and define it as a squared coordinates sum of this point reduced by the squared distance from a point to be determined:

$$
t_{s}=x_{s}^{2}+y_{s}^{2}+z_{s}^{2}-d_{s}^{2}
$$

where: $x_{s}, y_{s}, z_{s}$ - coordinates of the reference point/satellite $S\left(x_{s}, y_{s}, z_{s}\right)$, 
$d_{s}$ - distance of the reference point $S\left(x_{s}, y_{s}, z_{S}\right)$ from the determined point $Q\left(x_{Q}, y_{Q}, z_{Q}\right)$.

For satellite $i$, its reference point indicator is computed with regard to the determined point $Q\left(x_{Q}, y_{Q}, z_{Q}\right)$.

For GNSS (Oszczak, 2013) the equation can be expressed as follows:

$$
t_{i}=x_{i}^{2}+y_{i}^{2}+z_{i}^{2}-\rho_{i}^{2}
$$

where: $x_{i}, y_{i}, z_{i}-$ coordinates of the satellite $i$,

$\rho_{i}$ - pseudorange measured by the GNSS receiver to the satellite $i$.

\section{NEW ALGORITHM FOR DETERMINATION OF GNSS RECEIVER COORDINATES AND RECEIVER CLOCK ERROR FOR FIVE SATELLITES}

\subsection{Basic Equations.}

The author proposes a new GNSS positioning algorithm for five observed satellites. In the presented method five satellites are needed for the computation of GNSS receiver coordinates and the receiver clock error. The algorithm does not require the implementation of either the linearisation technique or the least squares method.

It is assumed that in the Cartesian coordinate system, the coordinates of five satellites are given: $1\left(x_{1}, y_{1}, z_{1}\right), 2\left(x_{2}, y_{2}, z_{2}\right), 3\left(x_{3}, y_{3}, z_{3}\right), 4\left(x_{4}, y_{4}, z_{4}\right), 5\left(x_{5}, y_{5}, z_{5}\right)$ on the basis of which the GNSS receiver coordinates at point $Q\left(x_{Q}, y_{Q}, z_{Q}\right)$ and the GNSS receiver clock error $b_{o}$ will be computed. Pseudoranges $\rho_{1}, \rho_{2}, \rho_{3}, \rho_{4}, \rho_{5}$ are measured with the GNSS receiver to each satellite and corrected due to ionosphere and troposphere errors and satellite clock errors. The unknowns $x_{Q}, y_{Q}, z_{Q}$ and the receiver clock error $b_{o}$ can be computed (Oszczak, 2013) using the formula:

$$
\boldsymbol{X}=\frac{1}{2} \boldsymbol{B} * \boldsymbol{A}^{-1}
$$

where:

$$
\boldsymbol{X}=\left[\begin{array}{l}
x_{Q} \\
y_{Q} \\
z_{Q} \\
\delta d
\end{array}\right]^{T} \quad \boldsymbol{B}=\left[\begin{array}{l}
\Delta t_{12} \\
\Delta t_{13} \\
\Delta t_{14} \\
\Delta t_{15}
\end{array}\right]^{T} \quad \boldsymbol{A}=\left[\begin{array}{llll}
\Delta x_{12} & \Delta y_{12} & \Delta z_{12} & \Delta \rho_{21} \\
\Delta x_{13} & \Delta y_{13} & \Delta z_{13} & \Delta \rho_{31} \\
\Delta x_{14} & \Delta y_{14} & \Delta z_{14} & \Delta \rho_{41} \\
\Delta x_{15} & \Delta y_{15} & \Delta z_{15} & \Delta \rho_{51}
\end{array}\right]
$$

$\boldsymbol{X}$ - matrix of unknown $x_{Q}, y_{Q}, z_{Q}$ and $\delta d$.

where: $\delta d-$ is a receiver clock bias expressed in metres, burdening all the pseudoranges in the system of equations, in which the location of point $Q\left(x_{Q}, y_{Q}, z_{Q}\right)$ is determined:

$$
\delta d=c b_{o}
$$

$b_{o}-$ the receiver clock error expressed in seconds,

$c$ - the speed of light,

$x_{Q}, y_{Q}, z_{Q}-$ the GNSS receiver coordinates.

The introduction of the additional fourth unknown $\delta \mathrm{d}$ in the system allows the coordinates of the determined point to be computed correctly.

The elements of the matrix $\mathbf{A}$ are as follows: 
where $i=2,3,4,5$.

$$
\begin{aligned}
\Delta x_{1 i} & =x_{i}-x_{1} \\
\Delta y_{1 i} & =y_{i}-y_{1} \\
\Delta z_{1 i} & =z_{i}-z_{1} \\
\Delta \rho_{i 1} & =\rho_{1}-\rho_{i}
\end{aligned}
$$

In the algorithm (chapter II.) proposed in this paper, each satellite's point indicator is computed with regard to the determined point $\mathrm{Q}\left(\mathrm{x}_{\mathrm{Q}}, \mathrm{y}_{\mathrm{Q}}, \mathrm{z}_{\mathrm{Q}}\right)$ :

$$
t_{i}=x_{i}^{2}+y_{i}^{2}+z_{i}^{2}-\rho_{i}^{2}
$$

The elements of the matrix $\mathbf{B}$ are as follows:

where $k=2,3,4,5$.

$$
\Delta t_{1 k}=t_{k}-t_{1}
$$

\subsection{Proof}

In the proposed algorithm, the corrected values of pseudoranges between the device sending a radio signal (satellite), and the device receiving the sent signal (GNSS receiver) are taken into account (Oszczak, 2012). There are five satellites in this solution and for each a separate reference point indicator is computed with regard to the GNSS receiver. It was assumed, as in the single point GPS algorithm (Parkinson and Spilker, 1996), in order to determine the unknowns $x_{Q}, y_{Q}, z_{Q}, \delta d$, the errors caused by alter alia ionospheric and tropospheric refraction and the satellite clock errors were eliminated from the measured pseudoranges.

After eliminating these errors we get the pseudorange values according to the formula:

$$
\begin{array}{r}
\rho_{i}=c t_{o}^{i}+\delta d-c t^{S} \\
i=1,2,3,4,5
\end{array}
$$

where:

$t_{o}^{i}-$ GNSS reception time of the signal from the $i-t h$ satellite in the GNSS receiver,

$t^{S}$ - time of sending a signal from satellite,

$\delta d-$ receiver clock error multiplied by the speed of light $c$,

$\delta d$ is defined as a constant systematic bias of the measurement burdening all the pseudoranges in the system of equations, from which the location of the GNSS receiver antenna - point $Q\left(x_{Q}, y_{Q}, z_{Q}\right)$ is determined (Oszczak, 2012).

The pseudoranges burdened by a systematic error $\delta \mathrm{d}$ can be expressed as follows:

where:

$$
\rho_{i}=d_{i}+\delta d
$$

$d_{i}$ - geometric distance of the point to be determined $Q\left(x_{Q}, y_{Q}, z_{Q}\right)$ to the $i-t h$ satellite, expressed by the formula:

$$
d_{i}^{2}=\left(x_{Q}-x_{i}\right)^{2}+\left(y_{Q}-y_{i}\right)^{2}+\left(z_{Q}-z_{i}\right)^{2}
$$

Thus, according to the formula (8): 


$$
t_{i}=x_{i}^{2}+y_{i}^{2}+z_{i}^{2}-\left(d_{i}+\delta d\right)^{2}
$$

After substituting the geometric distances $d_{i}$ to the equation (12) system of equations is obtained as follows:

$$
\begin{aligned}
& t_{1}=-x_{Q}^{2}-y_{Q}^{2}-z_{Q}^{2}+2 x_{Q} x_{1}+2 y_{Q} y_{1}+2 z_{Q} z_{1}-2 d_{1} \delta d-\delta d^{2} \\
& t_{2}=-x_{Q}^{2}-y_{Q}^{2}-z_{Q}^{2}+2 x_{Q} x_{2}+2 y_{Q} y_{2}+2 z_{Q} z_{2}-2 d_{2} \delta d-\delta d^{2} \\
& t_{3}=-x_{Q}^{2}-y_{Q}^{2}-z_{Q}^{2}+2 x_{Q} x_{3}+2 y_{Q} y_{3}+2 z_{Q} z_{3}-2 d_{3} \delta d-\delta d^{2} \\
& t_{4}=-x_{Q}^{2}-y_{Q}^{2}-z_{Q}^{2}+2 x_{Q} x_{4}+2 y_{Q} y_{4}+2 z_{Q} z_{4}-2 d_{4} \delta d-\delta d^{2} \\
& t_{5}=-x_{Q}^{2}-y_{Q}^{2}-z_{Q}^{2}+2 x_{Q} x_{5}+2 y_{Q} y_{5}+2 z_{Q} z_{5}-2 d_{5} \delta d-\delta d^{2}
\end{aligned}
$$

In the system of equations (13) the reference point indicator can be substracted from the others:

so/and:

$$
\begin{aligned}
& \Delta t_{12}=t_{2}-t_{1} \\
& \Delta t_{13}=t_{3}-t_{1} \\
& \Delta t_{14}=t_{4}-t_{1} \\
& \Delta t_{15}=t_{5}-t_{1}
\end{aligned}
$$

where:

$$
\begin{aligned}
& \Delta t_{12}=2 x_{Q}\left(\Delta x_{12}\right)+2 y_{Q}\left(\Delta y_{12}\right)+2 z_{Q}\left(\Delta z_{12}\right)+2 \delta d\left(\Delta d_{21}\right) \\
& \Delta t_{13}=2 x_{Q}\left(\Delta x_{13}\right)+2 y_{Q}\left(\Delta y_{13}\right)+2 z_{Q}\left(\Delta z_{13}\right)+2 \delta d\left(\Delta d_{31}\right) \\
& \Delta t_{14}=2 x_{Q}\left(\Delta x_{14}\right)+2 y_{Q}\left(\Delta y_{14}\right)+2 z_{Q}\left(\Delta z_{14}\right)+2 \delta d\left(\Delta d_{41}\right) \\
& \Delta t_{15}=2 x_{Q}\left(\Delta x_{15}\right)+2 y_{Q}\left(\Delta y_{15}\right)+2 z_{Q}\left(\Delta z_{15}\right)+2 \delta d\left(\Delta d_{51}\right)
\end{aligned}
$$

In this way, the second powers of the variables are eliminated, obtaining the solution (6) of this system, expressed in matrix form:

$$
\boldsymbol{X}=\frac{1}{2} \boldsymbol{B} * \boldsymbol{A}^{-1}
$$

$\boldsymbol{X}=\left[\begin{array}{l}x_{Q} \\ y_{Q} \\ z_{Q} \\ \delta d\end{array}\right]^{T} \quad \boldsymbol{B}=\left[\begin{array}{l}\Delta t_{12} \\ \Delta t_{13} \\ \Delta t_{14} \\ \Delta t_{15}\end{array}\right]^{T} \quad \boldsymbol{A}=\left[\begin{array}{llll}\Delta x_{12} & \Delta y_{12} & \Delta z_{12} & \Delta \rho_{21} \\ \Delta x_{13} & \Delta y_{13} & \Delta z_{13} & \Delta \rho_{31} \\ \Delta x_{14} & \Delta y_{14} & \Delta z_{14} & \Delta \rho_{41} \\ \Delta x_{15} & \Delta y_{15} & \Delta z_{15} & \Delta \rho_{51}\end{array}\right]$

where:

$$
\Delta \rho_{i 1}=\rho_{1}-\rho_{i}=d_{1}-d_{i}
$$

i.e. the formula (6), the validity of which we had to prove. However, satellites $1,2,3,4,5$ can be neither coplanar nor located on a common circle.

\subsection{Numerical Example}

In the presented example, a set of simulated data is given as follows:

1. In the Cartesian coordinate system at the moment of observation, the coordinates of five satellites are given:

as follows:

$$
1\left(x_{1}, y_{1}, z_{1}\right), 2\left(x_{2}, y_{2}, z_{2}\right), 3\left(x_{3}, y_{3}, z_{3}\right), 4\left(x_{4}, y_{4}, z_{4},\right), 5\left(x_{5}, y_{5}, z_{5}\right)
$$




$$
\begin{gathered}
x_{1}=28573624.909, y_{1}=176258.719, z_{1}=475886.493 \\
x_{2}=20534972.474, y_{2}=3620869.695, z_{2}=20821515.054 \\
x_{3}=13834909.426, y_{3}=9331764.237, z_{3}=24705373.313 \\
x_{4}=-1.8325015 .195, y_{4}=12831313.778, z_{4}=20831862.073 \\
x_{5}=-11441576.697, y_{5}=19817392.158, z_{5}=15998439.113
\end{gathered}
$$

2. Measured at the same epoch, pseudoranges $\rho_{1}, \rho_{2}, \rho_{3}, \rho_{4}, \rho_{5}$ corrected due to ionospheric and tropospheric errors and satellite clock errors are as follows:

$$
\begin{aligned}
& \rho_{1}=25573786.094 m \\
& \rho_{2}=23269991.712 m \\
& \rho_{3}=23527045.278 m \\
& \rho_{4}=29205487.559 m \\
& \rho_{5}=26129807.790 m
\end{aligned}
$$

The unknowns $x_{Q}, y_{Q}, z_{Q}$ and the receiver clock error $b_{o}$ can be computed in following manner. In the first step, a reference point indicator (8) is computed for each satellite with regard to the point $Q\left(x_{Q}, y_{Q}, z_{Q}\right)$ which is to be determined using MATLAB:

$$
\begin{aligned}
& t_{1}=159654526737584.50 \text { sq.m. } \\
& t_{2}=324075478316392.20 \text { sq.m. } \\
& t_{3}=332526379138345.90 \text { sq.m. } \\
& t_{4}=77987543295894.25 \text { sq.m. } \\
& t_{5}=93719451598142.75 \text { sq.m. }
\end{aligned}
$$

Using the measured pseudoranges the user position is determined and the results are shown as follows:

- the assumed user position is as follows:

$$
\begin{aligned}
& X=3461321.719 m \\
& Y=1276949.000 m \\
& Z=5185371.030 m
\end{aligned}
$$

- and the computed user position and receiver clock error correction using the new algorithm are:

$$
\begin{gathered}
x_{Q}=3461321.719 \mathrm{~m} \\
y_{Q}=1276949.000 \mathrm{~m} \\
z_{Q}=5185371.030 \mathrm{~m} \\
\delta d=59298.94819239155[\mathrm{~m}] \\
b_{o}=0.0001978[\mathrm{~s}]
\end{gathered}
$$

The method can be extended to the system of $n$-observational equations.

The generalised algorithms for $n$-number of observed satellites $(n>4)$ using $n$-indicator definitions have been developed and discussed by the author (Oszczak, 2013). 


\section{ALGORITHM FOR DETERMINATION OF GNSS RECEIVER COORDINATES AND RECEIVER CLOCK ERROR FOR TEN OBSERVED SATELLITES}

\subsection{Basic Equations}

The coordinates of ten satellites in the Cartesian coordinate system are assumed to be:

$1\left(x_{1}, y_{1}, z_{1}\right), 2\left(x_{2}, y_{2}, z_{2}\right), 3\left(x_{3}, y_{3}, z_{3}\right), 4\left(x_{4}, y_{4}, z_{4}\right), 5\left(x_{5}, y_{5}, z_{5}\right), 6\left(x_{6}, y_{6}, z_{6}\right), 7\left(x_{7}, y_{7}, z_{7}\right)$, $8\left(x_{8}, y_{8}, z_{8}\right), 9\left(x_{9}, y_{9}, z_{9}\right), 10\left(x_{10}, y_{10}, z_{10}\right)$,

on the basis of which the GNSS receiver coordinates at point $\mathrm{Q}\left(\mathrm{x}_{\mathrm{Q}}, \mathrm{y}_{\mathrm{Q}}, \mathrm{z}_{\mathrm{Q}}\right)$ and the GNSS receiver clock error $b_{0}$ will be computed. Pseudoranges $\rho_{1}, \rho_{2}, \rho_{3}, \rho_{4}, \rho_{5}, \rho_{6}, \rho_{7}, \rho_{8}, \rho_{9}, \rho_{10}$ are also given measured with the GNSS receiver to each satellite and corrected due to ionospheric and tropospheric errors and satellite clock errors. The unknowns $\mathrm{x}_{\mathrm{Q}}, \mathrm{y}_{\mathrm{Q}}, \mathrm{z}_{\mathrm{Q}}$ and the receiver clock error $b_{o}$ can be computed from the formula:

$$
\boldsymbol{X}=\frac{1}{2} \boldsymbol{B} * \boldsymbol{A}^{-1}
$$

where:

$$
\begin{gathered}
\boldsymbol{X}=\left[\begin{array}{l}
x_{Q} \\
y_{Q} \\
z_{Q} \\
\delta d
\end{array}\right]^{T} \boldsymbol{B}=\left[\begin{array}{c}
t_{\sum 34}-t_{\sum 12} \\
t_{\sum 56}-t_{\sum 12} \\
t_{\sum 78}-t_{\sum 12} \\
t_{\sum 910}-t_{\sum 12}
\end{array}\right]^{T} \\
\boldsymbol{A}=\left[\begin{array}{cccc}
\Delta x_{13}+\Delta x_{24} & \Delta y_{13}+\Delta y_{24} & \Delta z_{13}+\Delta z_{24} & \Delta \rho_{31}+\Delta \rho_{42} \\
\Delta x_{15}+\Delta x_{26} & \Delta y_{15}+\Delta y_{26} & \Delta z_{15}+\Delta z_{26} & \Delta \rho_{51}+\Delta \rho_{62} \\
\Delta x_{17}+\Delta x_{28} & \Delta y_{17}+\Delta y_{28} & \Delta z_{17}+\Delta z_{28} & \Delta \rho_{71}+\Delta \rho_{82} \\
\Delta x_{19}+\Delta x_{210} & \Delta y_{19}+\Delta y_{210} & \Delta z_{19}+\Delta z_{210} & \Delta \rho_{91}+\Delta \rho_{102}
\end{array}\right]
\end{gathered}
$$

The unknowns $x_{Q}, y_{Q}, z_{Q}$ and the receiver clock error $b_{o}$ can be computed in the following manner. For each two satellites it is possible to compute one reference two-point indicator (Oszczak, 2013). So in the first step, for ten satellites, five reference indicators for each pair of satellites are computed in regard to a point to be determined $Q\left(x_{Q}, y_{Q}, z_{Q}\right)$ :

$$
\begin{gathered}
t_{\sum 12}=x_{1}^{2}+y_{1}^{2}+z_{1}^{2}-\rho_{1}^{2}+x_{2}^{2}+y_{2}^{2}+z_{2}^{2}-\rho_{2}^{2} \\
t_{\sum 34}=x_{3}^{2}+y_{3}^{2}+z_{3}^{2}-\rho_{3}^{2}+x_{4}^{2}+y_{4}^{2}+z_{4}^{2}-\rho_{4}^{2} \\
t_{\sum 56}=x_{5}^{2}+y_{5}^{2}+z_{5}^{2}-\rho_{5}^{2}+x_{6}^{2}+y_{6}^{2}+z_{6}^{2}-\rho_{6}^{2} \\
t_{\sum 78}=x_{7}^{2}+y_{7}^{2}+z_{7}^{2}-\rho_{7}^{2}+x_{8}^{2}+y_{8}^{2}+z_{8}^{2}-\rho_{8}^{2} \\
t_{\sum 910}=x_{9}^{2}+y_{9}^{2}+z_{9}^{2}-\rho_{9}^{2}+x_{10}^{2}+y_{10}^{2}+z_{10}^{2}-\rho_{10}^{2}
\end{gathered}
$$

The elements of the matrix $\mathbf{A}$ are as follows:

$$
\begin{array}{ll}
\Delta x_{1 i}=x_{i}-x_{1} & \Delta x_{1 j}=x_{j}-x_{2} \\
\Delta y_{1 i}=y_{i}-y_{1} & \Delta y_{1 j}=y_{j}-y_{2}
\end{array}
$$




$$
\begin{array}{ll}
\Delta z_{1 i}=z_{i}-z_{1} & \Delta z_{1 j}=z_{j}-z_{2} \\
\Delta \rho_{i 1}=\rho_{1}-\rho_{i} & \Delta \rho_{j 2}=\rho_{2}-\rho_{j}
\end{array}
$$

where: $\quad i=3,5,7,9 \quad j=4,6,8,10$.

\subsection{Proof}

The reference two-point indicator (Oszczak, 2013) for satellite $i$ and $j$ is computed with regard to the determined point $Q\left(x_{Q}, y_{Q}, z_{Q}\right)$. For GNSS the equation is expressed as follows:

$$
t_{\sum i j}=x_{i}^{2}+y_{i}^{2}+z_{i}^{2}-\rho_{i}^{2}+x_{j}^{2}+y_{j}^{2}+z_{j}^{2}-\rho_{j}^{2}
$$

where:

$x_{i}, y_{i}, z_{i}-$ coordinates of satellite $i$,

$x_{j}, y_{j}, z_{j}$ - coordinates of satellite $j$,

$\rho_{i}$ - pseudorange measured with the GNSS receiver to the satellite $i$,

$\rho_{j}$ - pseudorange measured with the GNSS receiver to the satellite $j$.

It was assumed that measured pseudoranges were corrected due to ionospheric and tropospheric errors and satellite clock errors. Pseudoranges burdened by a systematic error $\delta \mathrm{d}$ can be written as:

$$
\rho_{i}=d_{i}+\delta d \quad \rho_{j}=d_{j}+\delta d
$$

where:

$d_{i}$-geometric distance of point $Q\left(x_{Q}, y_{Q}, z_{Q}\right)$ to be determined to the $i-t h$ satellite, expressed by the formula:

$$
d_{i}^{2}=\left(x_{Q}-x_{i}\right)^{2}+\left(y_{Q}-y_{i}\right)^{2}+\left(z_{Q}-z_{i}\right)^{2}
$$

$d_{j}$-geometric distance of point $\mathrm{Q}\left(\mathrm{x}_{\mathrm{Q}}, \mathrm{y}_{\mathrm{Q}}, \mathrm{z}_{\mathrm{Q}}\right)$ to be determined to the $j-$ th satellite, expressed by the formula:

$$
d_{j}^{2}=\left(x_{Q}-x_{j}\right)^{2}+\left(y_{Q}-y_{j}\right)^{2}+\left(z_{Q}-z_{j}\right)^{2}
$$

Thus, according to the formula (18):

where:

$$
t_{\sum i j}=\boldsymbol{K}_{i}+\boldsymbol{M}_{j}
$$

$$
\begin{aligned}
& \boldsymbol{K}_{i}=\left[x_{i}^{2}+y_{i}^{2}+z_{i}^{2}-\left(d_{i}+\delta d\right)^{2}\right] \\
& \boldsymbol{M}_{j}=\left[x_{j}^{2}+y_{j}^{2}+z_{j}^{2}-\left(d_{j}+\delta d\right)^{2}\right]
\end{aligned}
$$

For ten satellites there are five reference two-point indicators:

$$
\begin{aligned}
& t_{\sum 12}=x_{1}^{2}+y_{1}^{2}+z_{1}^{2}-\rho_{1}^{2}+x_{2}^{2}+y_{2}^{2}+z_{2}^{2}-\rho_{2}^{2} \\
& t_{\sum 34}=x_{3}^{2}+y_{3}^{2}+z_{3}^{2}-\rho_{3}^{2}+x_{4}^{2}+y_{4}^{2}+z_{4}^{2}-\rho_{4}^{2}
\end{aligned}
$$




$$
\begin{gathered}
t_{\sum 56}=x_{5}^{2}+y_{5}^{2}+z_{5}^{2}-\rho_{5}^{2}+x_{6}^{2}+y_{6}^{2}+z_{6}^{2}-\rho_{6}^{2} \\
t_{\sum 78}=x_{7}^{2}+y_{7}^{2}+z_{7}^{2}-\rho_{7}^{2}+x_{8}^{2}+y_{8}^{2}+z_{8}^{2}-\rho_{8}^{2} \\
t_{\sum 910}=x_{9}^{2}+y_{9}^{2}+z_{9}^{2}-\rho_{9}^{2}+x_{10}^{2}+y_{10}^{2}+z_{10}^{2}-\rho_{10}^{2}
\end{gathered}
$$

In the system of equations (22) reference two-point indicator $t_{\sum 12}$ can be subtracted from the others. In this way, the second powers of the variables can be eliminated and as a result there are four linear equations:

$$
\begin{aligned}
{\left[\mathrm{t}_{\Sigma 34}-\mathrm{t}_{\Sigma 12}\right.} & =\left[\begin{array}{llll}
2 x_{Q} & 2 y_{Q} & 2 z_{Q} & 2 \delta d
\end{array}\right] *\left[\begin{array}{l}
\Delta x_{13}+\Delta x_{24} \\
\Delta y_{13}+\Delta y_{24} \\
\Delta z_{13}+\Delta z_{24} \\
\Delta d_{31}+\Delta d_{42}
\end{array}\right] \\
{\left[\mathrm{t}_{\Sigma 56}-\mathrm{t}_{\Sigma 12}\right] } & =\left[\begin{array}{llll}
2 x_{Q} & 2 y_{Q} & 2 z_{Q} & 2 \delta d
\end{array}\right] *\left[\begin{array}{l}
\Delta x_{15}+\Delta x_{26} \\
\Delta y_{15}+\Delta y_{26} \\
\Delta z_{15}+\Delta z_{26} \\
\Delta d_{51}+\Delta d_{62}
\end{array}\right] \\
{\left[\mathrm{t}_{\Sigma 78}-\mathrm{t}_{\Sigma 12}\right] } & =\left[\begin{array}{llll}
2 x_{Q} & 2 y_{Q} & 2 z_{Q} & 2 \delta d
\end{array}\right] *\left[\begin{array}{l}
\Delta x_{17}+\Delta x_{28} \\
\Delta y_{17}+\Delta y_{28} \\
\Delta z_{17}+\Delta z_{28} \\
\Delta d_{71}+\Delta d_{82}
\end{array}\right] \\
{\left[\mathrm{t}_{\Sigma 910}-\mathrm{t}_{\sum 12}\right] } & =\left[\begin{array}{llll}
2 x_{Q} & 2 y_{Q} & 2 z_{Q} & 2 \delta d
\end{array}\right] *\left[\begin{array}{l}
\Delta x_{19}+\Delta x_{210} \\
\Delta y_{19}+\Delta y_{210} \\
\Delta z_{19}+\Delta z_{210} \\
\Delta d_{91}+\Delta d_{102}
\end{array}\right]
\end{aligned}
$$

The solution of this system is (16):

$$
\mathbf{X}=\frac{1}{2} \mathbf{B} * \mathbf{A}^{-1}
$$

where:

$$
\begin{gathered}
\boldsymbol{X}=\left[\begin{array}{l}
x_{Q} \\
y_{Q} \\
z_{Q} \\
\delta d
\end{array}\right]^{T} \quad \boldsymbol{B}=\left[\begin{array}{c}
t_{\sum 34}-t_{\sum 12} \\
t_{\sum 56}-t_{\sum 12} \\
t_{\sum 78}-t_{\sum 12} \\
t_{\sum 910}-t_{\sum 12}
\end{array}\right]^{T} \\
\boldsymbol{A}=\left[\begin{array}{cccc}
\Delta x_{13}+\Delta x_{24} & \Delta y_{13}+\Delta y_{24} & \Delta z_{13}+\Delta z_{24} & \Delta \rho_{31}+\Delta \rho_{42} \\
\Delta x_{15}+\Delta x_{26} & \Delta y_{15}+\Delta y_{26} & \Delta z_{15}+\Delta z_{26} & \Delta \rho_{51}+\Delta \rho_{62} \\
\Delta x_{17}+\Delta x_{28} & \Delta y_{17}+\Delta y_{28} & \Delta z_{17}+\Delta z_{28} & \Delta \rho_{71}+\Delta \rho_{82} \\
\Delta x_{19}+\Delta x_{210} & \Delta y_{19}+\Delta y_{210} & \Delta z_{19}+\Delta z_{210} & \Delta \rho_{91}+\Delta \rho_{102}
\end{array}\right]
\end{gathered}
$$

where: 


$$
\Delta \rho_{i 1}+\Delta \rho_{j 2}=\Delta d_{i 1}+\Delta d_{j 2}
$$

However, satellites $1,2,3,4,5,6,7,8,9,10$ can be neither coplanar nor located on a common circle.

\subsection{Numerical example for determination of GNSS receiver coordinates and receiver clock error for ten satellites.}

In the presented example, a set of simulated data is given as follows:

3.3.1. In the Cartesian coordinate system at the moment of observation, the coordinates of ten satellites are given:

$$
\begin{gathered}
1\left(x_{1}, y_{1}, z_{1}\right), 2\left(x_{2}, y_{2}, z_{2}\right), 3\left(x_{3}, y_{3}, z_{3}\right), 4\left(x_{4}, y_{4}, z_{4}\right), 5\left(x_{5}, y_{5}, z_{5}\right), 6\left(x_{6}, y_{6}, z_{6}\right), 7\left(x_{7}, y_{7}, z_{7}\right), 8\left(x_{8}, y_{8}, z_{8}\right) \\
9\left(x_{9}, y_{9}, z_{9}\right), 10\left(x_{10}, y_{10}, z_{10}\right) \\
x_{1}=28573843.196, y_{1}=186705.396, z_{1}=458504.029 \\
x_{2}=-13737297.587, y_{2}=23793697.380, z_{2}=440829.364 \\
x_{3}=135280.549, y_{3}=9472446.041, z_{3}=23550389.315 \\
x_{4}=-17629491.025, y_{4}=10178391.389, z_{4}=20326540.307 \\
x_{5}=21444538.037, y_{5}=9999752.312, z_{5}=16543394.085 \\
x_{6}=-8952698.519, y_{6}=24597337.024, z_{6}=12187985.352 \\
x_{7}=13576242.929, y_{7}=20905580.826, z_{7}=11605617.387 \\
x_{8}=2107612.980, y_{8}=24090126.595, z_{8}=19555410.293 \\
x_{9}=-10553478.506, y_{9}=4921167.847, z_{9}=26114803.717 \\
x_{10}=-290863.203, y_{10}=5550000.536, z_{10}=26104633.518
\end{gathered}
$$

3.3.2. Measured at the same epoch, pseudoranges $\rho_{1}, \rho_{2}, \rho_{3}, \rho_{4}, \rho_{5}, \rho_{6}, \rho_{7}, \rho_{8}, \rho_{9}, \rho_{10}$ corrected due to ionospheric and tropospheric errors and satellite clock errors are as follows:

$$
\begin{gathered}
\rho_{1}=25449152.282 \mathrm{~m} ; \rho_{2}=28710125.200 \mathrm{~m} ; \rho_{3}=22512803.080 \mathrm{~m} \\
\rho_{4}=27609639.021 \mathrm{~m} ; \rho_{5}=22920682.547 \mathrm{~m} ; \rho_{6}=27338791.883 \mathrm{~m}, \\
\rho_{7}=22881688.771 \mathrm{~m}, \rho_{8}=26984600.739 \mathrm{~m} \\
\rho_{9}=25643828.772 \mathrm{~m}, \rho_{10}=21830588.390 \mathrm{~m} .
\end{gathered}
$$

The unknowns $x_{Q}, y_{Q}, z_{Q}$ and the receiver clock error can be computed according to the formula (16).Using the measured pseudoranges, the user position is determined and results are shown below:

the assumed user position is as follows:

$$
\begin{aligned}
& X=3600893.146 m \\
& Y=1414800.819 m
\end{aligned}
$$




$$
Z=5053752.000 \mathrm{~m}
$$

the computed user position and receiver clock error correction using the new algorithm are:

$$
\begin{gathered}
x_{Q}=3600893.146 \mathrm{~m} \\
y_{Q}=1414800.819 \mathrm{~m} \\
z_{Q}=5053752.000 \mathrm{~m} \\
\delta d=2725706.432709098[\mathrm{~m}] \\
b_{o}=\frac{\delta d}{c}=0.00009091978[\mathrm{~s}]
\end{gathered}
$$

\section{DISCUSSION AND CONCLUSIONS}

The author developed the novel algorithms for GNSS position determination using the proposed mathematical definitions of reference point indicators and reference two-point indicators. The algorithms for five and ten satellites, presented by the author, do not require linearisation of the observation equations and the least squares method. It was assumed that pseudoranges measured by the GNSS receiver to each satellite are corrected due to ionospheric and tropospheric errors and satellite clock errors. It is necessary to eliminate these errors to compute the correct value of the systematic bias $\delta d$ which burden observations in the system of equations, in which the location of the point is determined. This is a disadvantage of the proposed method. The introduction of the unknown $\delta d$ in the system of equations makes it possible to compute the correct coordinates of the point to be determined without regard to the value of the systematic error of the GNSS receiver clock. The presented algorithms should be further tested on the basis of real measurements and in other domains of positioning and navigation. The generalised algorithms for $n$-number of measured distances $(n>4)$ using reference two-point indicator have been developed by the author.

\section{REFERENCES}

B. Hofmann-Wellenhof, H. Lichtenegger, E. Wasle, "Point positioning with code ranges" in GNSS Global Navigation Satellite Systems, Ed. SpringerWienNewYork, 2008, Austria, pp. 161-163.

J. Klobuchar, "Design and characteristics of the GPS ionospheric time - delay algorithm for single frequency" in Position Location and Navigation Symposium., Las Vegas, Nevada, 1986, November 4-7, pp. 280-286.

H.S. Hopfield, "Two-quartic tropospheric refractivity profile for correcting satellite data" in Journal of Geophysical Research, 1969, Vol. 74, No. 18, pp. 4487-4499.

G. Strang, K. Borre, "Receiver position from code observations" in Linear Algebra, Geodesy and GPS,1997, Ed. Wellesley-Cambridge, MA, pp. 460-472.

J.B. Tsui, "Basic equations for finding user position" in Fundamentals of Global positioning system receivers. A software approach. Ed. John Wiley \& Sons, 2000, pp. 10-11

M. Paonni, M. Anghileri, J.A. Avila-Rodriguez S. Wallner, B. Eissfeller "Performance assesment of GNSS signals in terms of time to first fix for cold, warm and hot start" in Proc. of the ION ITM 2010, San Diego, CA, January 25-27. 
S. Bancroft, "An algebraic solution of the GPS equations" in IEEE Transactions on Aerospace and Electronic Systems, 1991, Vol. 30, No. 4, pp. 1021-1030.

J.S. Abel, J.W. Chaffee "Existence and uniqueness of GPS solutions" in IEEE Transactions on Aerospace and Electronic Systems, 1991, Vol. 27, No. 6, pp. 952-956.

J.W. Chaffee, J.S. Abel "On the exact solutions of pseudorange equations" in IEEE Transactions on Aerospace and Electronic Systems, 1991, Vol. 30, No. 4, pp. 1021-1030.

E.W. Grafarend, J.A. Chan "A closed-form solution of the nonlinear pseudo-ranging equations" in Artificial Satellites Planetary Geodesy, 1996, No. 28, pp. 133-147.

A. Kleusberg, "Die direkte Losung des raumlichen hyperbelschnitts" in Zeitschrift fur vermessungswesen, 1994, Vol. 119, No. 4, pp. 188-192.

S. Hausbrandt "Auxiliary algorithm" in "Rachunek wyrownawczy i obliczenia geodezyjne", Ed. PWN, 1970, Warsaw, Poland, pp. 530-540.

P. Misra, P. Enge, "Code phase measurements" in Global Positioning System. Signals, measurements, and performance", Ed. Ganga-Jamuna Press, 2006, Massachusetts, pp. 148-155.

B. Oszczak "New algorithm for GNSS positioning using system of linear equations", in Proc. of the ION GNSS+2013, Nashville, Tennessee, September 17-20.

B. Oszczak "Algorytm wyznaczania wspolrzednych punktu i dodatkowej niewiadomej z zastosowaniem ukladow rownan liniowych (Algorithm for determination of the coordinates and additional unknown using linear equations)", Scientific Bulletin, Ed. Air Force Academy, 2012, No. 2, Deblin, pp. 163-167.

B.W.Parkinson, J.J. Spilker Jr., "GPS error analysis" in Global Positioning System. Theory and Applications. 1996, Ed. American Institute of Navigation and Astronautics, Washington, Vol 1, pp. 469-483.

B. Oszczak, E. Sitnik "The Algorithm for Determining the Coordinates of a Point in ThreeDimensional Space by Using the Auxiliary Point". Artificial Satellites. Volume 48, Issue 4, Pages 141-145, ISSN (Online) 2083-6104, ISSN (Print) 0208-841X, DOI: 10.2478/arsa-2013-0012, December 2013.

Received: 2014-02-21,

Reviewed: 2014-03-11, by W. Kosek,

Accepted: 2013-03-12. 